\title{
What Factors Affect the Usefulness of Online Reviews? An Empirical Study Based on Signaling Theory
}

\author{
Song Sujuan*, Peng Wei, and Cai Limei \\ Business School, Sichuan Agricultural University, Chengdu, China
}

Keywords: Online reviews; Usefulness of online reviews; Signaling theory

\begin{abstract}
Online reviews play an increasingly important role in consumers' purchasing decisions. How to identify high-quality online reviews has become a research hot-spots. Based on the signaling theory, this paper explores the factors that affect the usefulness of online reviews from three aspects: the signals related to reviewers, the signals related to review characteristics, and the signals related to feedback. Through the empirical analysis of 2305 valid samples in JD.com, it is found that additional reviews, review videos, number of review replies and number of pictures have a significant impact on the usefulness of online reviews, and the most obvious impact of them is the number of review replies. But the reviewer's credit rating have not significant effect on the usefulness of online review. The research's findings are of great practical significance to the management of e-commerce platforms, the improvement of merchant products, and the consumers' purchasing decisions.
\end{abstract}

\section{Introduction}

With the vigorous development of the Internet, people's consumption has gradually changed from the traditional mode of passively receiving information to the online shopping mode of actively searching for information. Channel Advisor's survey found that $90 \%$ of consumers browse online reviews before purchasing a product, and 83\% of them think that their final decision will be affected by online reviews [1]. Online reviews have become an important factor in corporate Internet marketing or brand management, as well as an important source of information for consumers to make decisions.

At present, most domestic e-commerce websites allow and encourage consumers to express their views on the goods or services they have purchased. In addition, social networking sites have mushroomed in recent years, also providing a platform for consumers to post reviews [2]. With the popularity of online reviews, consumers can easily find information about products to help them make decisions. However, this also brings another problem, that is, the overload of the number of reviews, the negative impact of economic returns on reviews, etc., which makes it difficult for consumers to find useful information. In response to this phenomenon, many e-commerce platforms set up a vote next to a single review on whether it is useful for consumers. But the useful voting mechanism does not solve all problems: for unpopular commodities, the useful voting mechanism loses its effect. Therefore, research on the usefulness of online reviews is necessary.

In view of this, on the basis of previous literature and signal transmission theory, this study analyzes the factors that affect the usefulness of online review from three aspects: the signal related to reviewers, the signal related to review characteristics and the signal related to feedback. This study differs from previous studies in that: (1) No longer limited to the influence of the presence or absence of pictures on the usefulness of online reviews, this paper also explores the impact of the number of pictures and review videos on the usefulness of online reviews. (2) from a new perspective, based on the theory of signal transmission, this paper studies how various factors affect the usefulness of online reviews. (3) a new explanation is given in this paper for the inconsistent results of previous studies, which further enriches the exploration of the usefulness of online reviews. 


\section{Literature Reviews and Theoretical Basis}

\subsection{Research on the usefulness of online reviews}

Chatterjee [3] first proposed the concept of usefulness of reviews. Mudambi\&Schuff (2010) put forward the concept of usefulness of online reviews from the perspective of perceived value, that is, the extent to which this review information helps consumers to make purchasing decisions [4]. At present the existing literature research on the usefulness of reviews mainly analyzes the influencing factors of the usefulness of reviews from two perspectives: the characteristics of reviewers and the characteristics of reviews.

From the perspective of reviewer characteristics, Froman $C$, when exploring the characteristics of reviewers, proposed to treat reviewers as sellers of reviews, and certain characteristics of reviewers will affect consumer acceptance of reviews [5]. Liu (2015) used a combination of quantitative and qualitative methods to explore the usefulness of online reviews, and concluded that the higher the reviewer's credit rating, the more useful the review is [6]. Based on the theory of signaling, this paper will explore the influence of reviewers' credit rating on the usefulness of reviews from a new perspective.

From the perspective of review characteristics, Mitchell A (1986) proposed that picture information has a greater impact on consumers than text information [7]. The reason is that readers can not only indirectly change their views on products through pictures, but also reviewers' emotional responses can directly affect readers' attitude to the product. Xu (2015) studied the impact of the presentation form of reviews on the usefulness of online reviews, and concluded that reviews containing videos are more useful to consumers than traditional text-type reviews [8]. Zhang (2016) proposed that reviewers supplement and improve previous reviews and publish their new feelings, which can increase readers' attention to the reviews [9]. However, few researches have paid much attention to the impact of the number of pictures and review videos on the usefulness of online reviews. In addition, the research on the usefulness of online reviews mainly focuses on the first review after consumers receive the product. While the research on the usefulness of additional reviews is still scarce.

\subsection{Signaling theory}

Signaling theory, originated from information economics, was proposed by Spence $\mathrm{M}$ at the end of the 20th century, Which is used to explain the situation of information asymmetry. At present, it has developed into a theoretical framework including signal sender, signal receiver, feedback and other elements [10]. In the virtual network community, buyers and sellers are faced with information asymmetry. In order to better explain the process by which consumers determine whether reviews are useful, the theory of signal transmission is introduced into the research. According to this theory, the signal sender (reviewer) sends a signal to the signal receiver (review reader), which to some extent can make up for the defect of information asymmetry related to commodities.

Signals can be divided into: signals related to reviewers, signals related to review characteristics and signals related to feedback [11]. The signals related to the reviewers are generally provided by the e-commerce platform based on the past behavior of the reviewers. The reviewers need to pay a lot of costs to obtain such signals. However, the signals related to reviews are usually included in the review text, and the cost for consumers to send such signals is low. review readers, as the signal receiver, hope to get useful information through reading reviews, so as to help them make the final decision. At the same time, the review reader can also give feedback to the signal sender in the form of review reply. 


\section{Research Frame Work and Hypotheses}

\subsection{Signals related to reviewers}

\subsubsection{Reviewer credit rating}

Information source is the most critical factor that affects the credibility of information. In the reviews of goods or services, the signal sent by the reviewer is used as the information source of the review. Its characteristics have an important impact on the usefulness of the online review. However, in virtual online communities, there is a weak correlation between potential consumers and reviewers. They lack understanding of reviewers' preferences, personal image and professional level, etc., which makes it difficult to identify the credibility of reviews. Juliet (2017) believes that reviewers with higher credit ratings and more product-related knowledge tend to have rich purchasing experience, which can provide more comprehensive information for review readers and help consumers make decisions, so their reviews are considered more useful [12]. The characteristics of the reviewer include many factors such as gender, age, occupation, credit rating and geographic location. However, JD.com only provides the option of credit rating [13]. Therefore, this article only studies the influence of the reviewer's credit rating on the usefulness of online reviews. Based on this, this paper proposes the following assumptions:

Hypothesis 1 (H1). The credit rating of reviewers has a positive impact on the usefulness of online reviews.

\subsection{Signals related to review characteristics}

\subsubsection{Review picture}

Different from the traditional consumption mode, in the online purchasing mode, consumers cannot personally feel the quality of goods, so they often obtain the product information more intuitively through the pictures provided by merchants and uploaded by reviewers. Pictures of products or services provided by merchants are mostly processed and beautified, which cannot objectively reflect the characteristics of commodities. And the pictures taken by electronic devices when consumers make reviews can objectively reflect the real situation of products or services, thereby reducing consumers' uncertainty about products. Min (2017) in exploring the influencing factors of the usefulness of online reviews, based on the dual coding of cognitive psychology, concluded that reviews with pictures are an important factor affecting the usefulness of online reviews [14]. At present, many studies have confirmed that review pictures have a positive effect on review usefulness, but there is little research on the impact of the number of pictures on review usefulness. Based on this, the following hypotheses are proposed in this study:

Hypothesis 2a (H2a). The more pictures in a review, the more useful the review is.

\subsubsection{Additional review}

In the early stage of e-commerce, the review system of shopping websites only provides consumers with the function of review once. With the improvement of the shopping system of e-commerce platforms, many shopping websites allow reviewers to make additional reviews on purchased products. However, most of the previous researches on the usefulness of online reviews are focused on one-time reviews, and a few scholars will pay attention to the additional reviews of consumers. When consumers read product reviews, they will perceive the usefulness of the first review and the additional review as a whole. Zhang (2016) believes that the additional reviews are supplementary explanations or revisions to the first reviews, which will attract the attention of potential consumers [9]. Therefore, this paper proposes the following assumptions:

Hypothesis $2 \mathrm{~b}(\mathrm{H} 2 \mathrm{~b})$. Additional reviews have a positive impact on the usefulness of online reviews.

\subsubsection{Review video}

Review video is a new element added by shopping websites in recent years. The video in the 
review contains comprehensive product information, which is more persuasive to consumers. Susan and David (2010) proposed that the depth of information can strengthen consumer confidence in the product and thus facilitate the entire decision-making process [4]. The depth of review information is not only reflected in the additional review, review pictures, etc., but also can provide further evidence for the reviewers' views through the review video. Compared with the reviews only containing words, the signals sent by video are undoubtedly more authentic and appealing, and more likely to arouse consumers' curiosity about products. Based on this, this paper proposes the following assumptions:

Hypothesis 2c(H2c). Video-based signals have a positive impact on the usefulness of online reviews.

\subsection{Signals related to feedback}

Review readers can not only obtain product or service information by receiving signals from reviewers, but also can provide feedback on signals in the form of replies.Wang (2016) proposed that the number of replies has a positive impact on the usefulness of online reviews based on the moderating effect of commodity types [15]. In addition, consumer responses to reviews can also help other review readers who have the same problem. The more review replies there are, the more attention there is to the review, which also reflects the usefulness of the online review to some extent. Therefore, it is hypothesized that:

Hypothesis H3(H3). The more replies in a review, the more useful the review is.

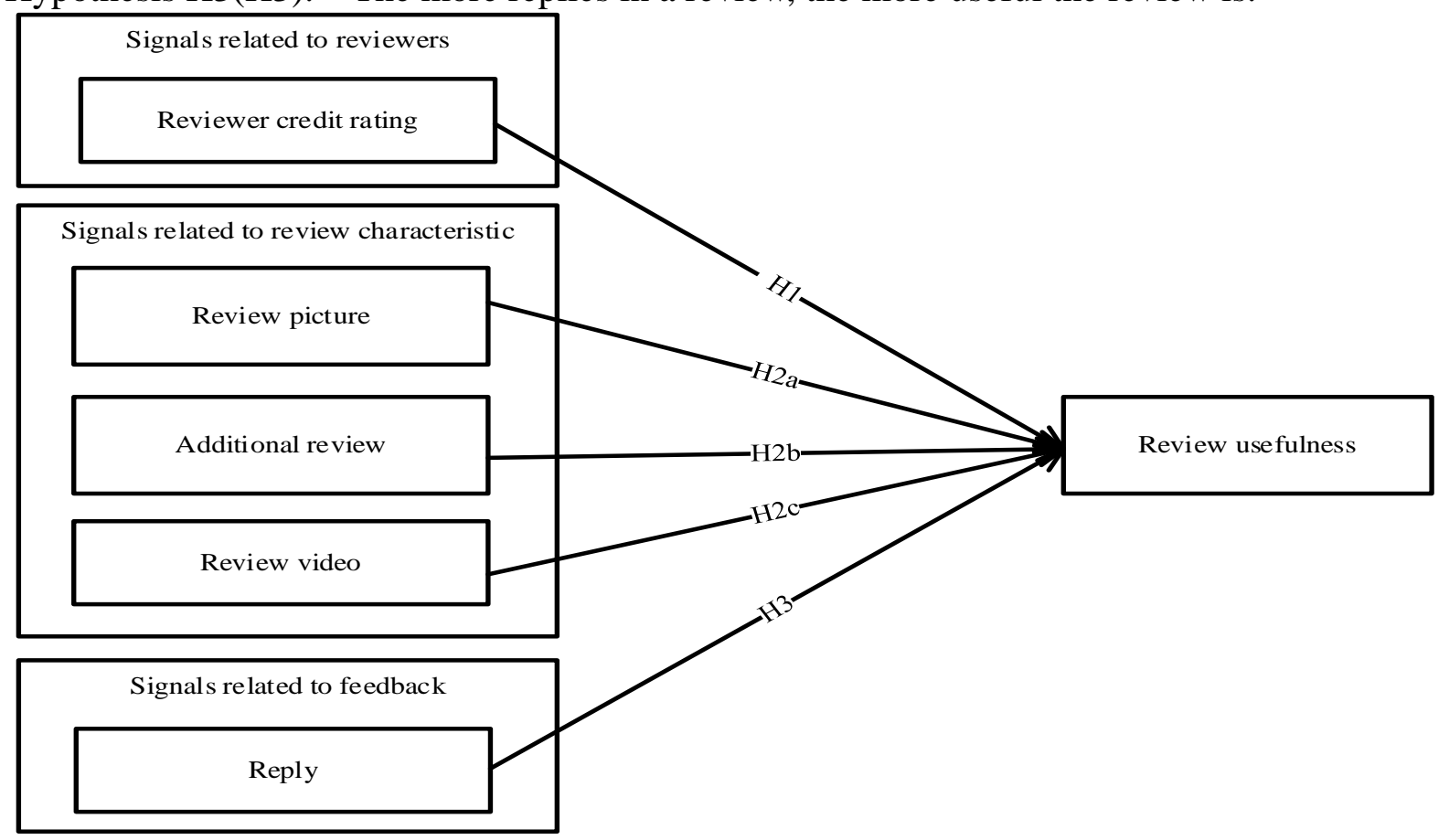

Figure 1 The proposed research model.

Figure 1 is the research model proposed in this paper. The model shows that reviewer credit rating, number of pictures, additional reviews, review videos, and review replies have a direct impact on the usefulness of online reviews.

\section{Research data ana variable}

\subsection{Data collection and preprocessing}

In this study, python crawler system software compiled by ourselves was used to crawl some consumer review data of selected products on the JD.com, totaling 4068 pieces. The display page of product information on the website of JD.com contains reviews on products. We captured the reviewer credit rating, number of pictures, additional reviews, review video, usefulness votes for each review on three products: tablet, computer, and mobile. In order to ensure the validity of the 
data, we first remove the reviews with the content of "this user has not evaluated", then remove the reviews of the discontinued products, and finally get 2305 pieces of valid data.

\subsection{Research variables}

The hypothesis indicates that the signal related to the reviewer mainly refers to the reviewer's credit rating. In JD.com, the system will give consumers "Plus" members for free because of their credit points, so the credit rating code of reviewers with JD.com "plus" members is 1 , otherwise 0 . The signals related to reviews mainly include review pictures, additional reviews, and review videos. In this study, reviews containing additional reviews or videos are coded as 1 , otherwise coded as 0 . Review pictures are measured by the number of pictures contained in a review. Feedback-related signals are measured by the number of replies in a review. The dependent variable was measured by the number of useful votes obtained from the reviews. The specific names of the variables are shown in Table 1.

Table 1 Variable definition.

\begin{tabular}{|c|c|c|}
\hline Variable category & Variable name & Definition \\
\hline Dependent variable & Helpful & Review usefulness \\
\hline the signals related to reviewers & Credit rating & Whether or not reviewer is a”Plus" members. \\
\hline $\begin{array}{c}\text { Signals related to review } \\
\text { characteristics }\end{array}$ & Picture & Number of picture in a review \\
\cline { 2 - 3 } & Additional review & Whether or not review contains additional review \\
\cline { 2 - 3 } & video & Whether or not review contains a video \\
\hline Signals related to feedback & Reply & Review replies \\
\hline
\end{tabular}

Descriptive statistics of the variables are shown in Table 2. As can be seen from table 2, the number of pictures in different reviews varies greatly, with the maximum reaching 10 and the minimum 0 . Therefore, it is a reasonable choice to explore the number of pictures in reviews.

Table 2 Descriptive statistics for the variable.

\begin{tabular}{|c|c|c|c|c|c|}
\hline variable & $\mathrm{N}$ & Mean & Std & Minimum & Maximum \\
\hline Helpful & 2305 & 4.75 & 24.987 & 0 & 731 \\
\hline Credit rating & 2305 & 0.33 & 0.471 & 0 & 1 \\
\hline Picture & 2305 & 2.77 & 2.577 & 0 & 10 \\
\hline Additional review & 2305 & 0.06 & 0.244 & 0 & 1 \\
\hline Video & 2305 & 0.35 & 0.477 & 0 & 1 \\
\hline Reply & 2305 & 2.07 & 11.024 & 0 & 266 \\
\hline
\end{tabular}

\section{Empirical results and analysis}

\subsection{Setting of regression model}

In this study, SPSS 22.0 software is used for linear regression analysis of the collected data. Combined with the hypothesis of previous studies, the overall regression model of this paper is:

Helpful $=\beta 0+\beta 1^{*}$ Credit rating $+\beta 2 *$ Picture $+\beta 3^{*}$ Additional Review $+\beta 4 *$ Video $+\beta 5^{*}$ Reply

\subsection{Empirical analysis}

The correlation between variables is described in Table 3. It can be seen from the table 3 that there is a significant correlation between the credit rating of reviewers, the number of pictures, additional reviews, review videos, review replies and the number of usefulness votes. In addition, the correlation coefficient between independent variables is less than 0.8 , indicating that there is no strong correlation between independent variables, so regression analysis can be carried out for all variables.

This paper uses a hierarchical regression method to test the impact of each variable on the usefulness of online reviews. Table 4 shows the results of the regression model, and R-squared is used to judge the fitting effect of each model. Finally, with the addition of new variables, the model 
has improved and the fitting degree is better.

Table 3 Correlation coefficient.

\begin{tabular}{|c|c|c|c|c|c|c|}
\hline variable & Helpful & Credit rating & Picture & Additional review & Video & Reply \\
\hline Helpful & 1.000 & -0.013 & 0.099 & 0.115 & 0.070 & 0.770 \\
\hline Credit rating & -0.013 & 1.000 & 0.025 & -0.017 & 0.013 & -0.004 \\
\hline Picture & 0.099 & 0.025 & 1.000 & 0.068 & -0.590 & 0.110 \\
\hline Additional Review & 0.115 & -0.017 & 0.068 & 1.000 & -0.043 & 0.096 \\
\hline Video & 0.070 & 0.013 & -0.590 & -0.043 & 1.000 & 0.048 \\
\hline Reply & 0.770 & -0.004 & 0.100 & 0.096 & 0.048 & 1.000 \\
\hline
\end{tabular}

Table 4 Results of regression analysis.

\begin{tabular}{|c|c|c|c|c|}
\hline Variable type & Variable abbreviation & Model 1 & Model 2 & Model 3 \\
\hline Signals related to reviewers & Credit rating & -0.013 & -0.019 & -0.012 \\
\hline \multirow{2}{*}{$\begin{array}{c}\text { Signals related to review } \\
\text { characteristics }\end{array}$} & Picture & & $0.209^{* * *}$ & $0.052^{* *}$ \\
\cline { 2 - 5 } & Additional review & & $0.108^{* * *}$ & $0.041^{* *}$ \\
\cline { 2 - 5 } & Video & & $0.199^{* * *}$ & $0.066^{* * *}$ \\
\hline Signals related to feedback & Reply & & & $0.757^{* * *}$ \\
\hline \multicolumn{2}{|c|}{ R-squared } & 0.00018 & 0.048 & 0.598 \\
\hline \multicolumn{2}{|c|}{$\Delta$ R-squared } & 0.00018 & 0.0478 & 0.55 \\
\hline
\end{tabular}

Note: ${ }^{*} \mathrm{P}<0.1 .^{* *} \mathrm{P}<0.05 .{ }^{* * *} \mathrm{P}<0.001$.

The detailed results of the regression model are shown in Table 4. Model 1 adds signals related to reviewers, and the results show that the reviewer's credit rating has no significant effect on the usefulness of online reviews $(\beta 1=-0.013, \mathrm{p}=0.520)$. One possible reason is that "Plus" member is not a complete guide to judge the credit rating of reviewers. In JD.com, some reviewers are given "plus" members because of their high credit rating. However, in order to enjoy the preferential treatment of "Plus" members, some consumers obtain it by means of charging money. The existence of this method has led to the assumption that $\mathrm{H} 1$ is not valid.

On the basis of Model 1, Model 2 continues to add reviews-related signals, including the number of pictures, additional reviews, and review videos. Among them, the regression coefficients of the number of pictures $(\beta 2=0.209, \mathrm{p}<0.001)$, additional reviews $(\beta 3=0.108, \mathrm{p}<0.001)$, and review videos $(\beta 4=0.199, \mathrm{p}<0.001)$ all passed the significance test. Hypothesis H2a, H2b, H2c are supported. Therefore, the platform can take some incentives to encourage consumers to post reviews containing pictures, additional reviews, and videos, thereby enhancing consumers' perception of the usefulness of online reviews, such as giving material rewards to consumers who post high-quality reviews, or publishing satisfaction requested reviewers can be drawn.

On the basis of model 2, model 3 continues to add feedback-related signals, that is, review replies. The results show that the regression coefficient $\beta 5$ of review replies is 0.757 and significant at the level of 0.001 , indicating that the more review replies, the more useful review is. In addition, with the addition of the review replies in the model, the fitting degree has changed greatly, and the same conclusion has been obtained through multiple tests of different numbers of data, which shows that review replies has the most obvious impact on the usefulness of reviews. Hypothesis H3 is supported.

In order to ensure the stability of the regression results, this study further made a colinear diagnosis of the variables. The test results are shown in Table 5. In the experiment, the variance expansion factor between the variables is not more than 10 , which shows that there is no serious collinearity between the variables, and further proves the stability of the results. 
Table 5 The results of VIF and tolerance in the regression model.

\begin{tabular}{|c|c|c|}
\hline Variable & VIF & Tolerance \\
\hline Grade & 1.002 & 0.998 \\
\hline Picture & 1.583 & 0.632 \\
\hline Additional C & 1.013 & 0.987 \\
\hline Video & 1.565 & 0.639 \\
\hline Reply & 1.042 & 0.960 \\
\hline
\end{tabular}

\section{Conclusions}

\subsection{Research conclusion}

Based on the theory of signal transmission, this paper explores its influence on the usefulness of online reviews from three aspects: the signal related to reviewers, the signal related to review characteristics and the signal related to feedback. On this basis, this paper selects a number of commodity reviews on JD.com and uses second-hand data to conduct an empirical test on the theoretical model. The results show that among the signals related to reviewers, the reviewer's credit rating has no significant effect on the usefulness of the online reviews. Among the signals related to reviews, the more pictures, additional reviews and video reviews are more useful. In the feedback-related signals, the number of review replies has a significant positive effect on the usefulness of online reviews.

\subsection{Research significance}

This research has the following contributions, mainly including theoretical contributions and practical contributions.

\subsubsection{Theoretical significance}

Most previous studies used information adoption models, elaboration likelihood models to construct influential factor models for online review usefulness. This research, based on the signal transfer theory, aims to explore the factors that affect the usefulness of online reviews from a new perspective. In addition, this study introduces feedback-related signals, instead of focusing only on reviewer characteristics and review characteristics, which is also different from previous studies.

\subsubsection{Practical significance}

From the perspective of the merchant, the conclusions of this study can help merchants improve the product itself and provide products that better meet consumer requirements, thereby achieving the goal of increasing sales. From the perspective of e-commerce platform, the conclusion of this study can help the platform to improve the shopping system, enhance consumers' preference for the platform, and finally achieve the goal of improving the popularity of the website. From the perspective of consumers, the conclusions of this study can reduce the cognitive cost of consumers, eliminate the uncertainty of consumers on the goods, and help them make purchasing decisions.

\subsection{Research limitations and possible future research directions}

There are also some limitations in this study. First, when exploring the signals related to reviewers, this study only judges the reviewer's credit rating by whether the reviewer in JD.com has "plus" members, and it is limited to one website, so it can't get the impact intensity of the reviewer's credit rating on the usefulness of online reviews. In future research, on the one hand, multiple indicators can be added to define the credit rating of the reviewer, and on the other hand, cross platform research can be carried out, which is also a less concerned aspect in this field at present. Second, this paper respectively explores the impact of the number of pictures and whether the reviews contain video on the usefulness of online reviews, but only makes an independent analysis of them, and does not explore the impact of their commonness and characteristics on the usefulness of online reviews. Future research can improve this aspect. In addition, future research can be 
carried out across fields, such as the combination of network nodes of reviewers and mathematical modeling.

\section{References}

[1] Mathur K. Sharma A. (2015). A Study of Online Shopping Habits of Consumers in India. International Journal on Customer Relations.

[2] Shiyang Gong, Xia Liu, Ping Zhao. (2013) How Does Online Consumer Reviews Affect Product Sales? Empirical Research Based on Online Book Reviews. China Soft Science Magazine, (6):176-188.

[3] CHATTER JEEP. Online Reviews: Do Consumers Use Them?.Advances in Consumer Research, 2001, 28(1):129-133.

[4] Mudambi S. M. Schuff D. (2010). What Makes a Helpful Online Reveiw? A Study of Customer Reviews on Amazon. Com. MIS Quarterly, 34(1): 185-200.

[5] Forman C. Ghose A. Wiesenfeld B. (2008). Examining the Relationship Between Reviews and Sales: The Role of Reviewer Identity Disclosure in Electronic Markets. Information Systems Research, 19(3): 291-313.

[6] Zhiwei Liu, Park, Sangwon. (2015). What makes a useful online review? Implication for travel product websites. Tourism Management, (47):140-151.

[7] Mitchell A. A. (1986). The Effect of Verbal and Visual Components of Advertisements on Brand Attitudes and Attitude Toward the Advertisement. Journal of Consumer Research, 13(1): 12-24.

[8] Xu P., Chen L, Santhanam R. (2015). Will Video Be the Next Generation of E-commerce Product Reviews? Presentation Format and the Role of Product Type. Decision Support Systems, 73: 85-96

[9] Huiyang Zhang, Zongwei Li (2016). A Study on the Influencing Factors of the Usefulness of Online Reviews: Moderating Role Based on Product Type. Business Review, 28(10).

[10] Spence M. (1973). Job Market Signaling. The Quarterly Journal of Economics, 87 (3) : 355-374.

[11] Li. Zhao, Zhijie. (2019). Research on the Influential Factors of the Usefulness of Online Reviews Based on Signaling Theory. Journal of Modern Information.

[12] Liye Zhu. Yuan, Denghua. Zhang, Jinyi. (2017). The Influence of Online Users' Review Quality and Reviewer's Credit Rating on Consumers' Purchase Intention: The Moderating Effect of Product Involvement. Business Review, 29(2).

[13] Yanfeng Zhang, Li He, Peng Lihui, et al. An empirical study on the influencing factors of the timeliness characteristics of online users' reviews. Journal of Modern Information, 39(01):61-70+78.

[14] Qingfei Min. Qin, Liang. Zhang, Keling. (2017). Research on Factors Affecting the Usefulness of Online Reviews. Business Review, 29(10):95-107.

[15] Zhishen Wang. Huiyi Li, Rui Sun, et al. (2016). A Study on the Influencing Factors of the Usefulness Voting of Online Reviews: Moderating Role Based on Product Type. Business Review, 28(7):143-153. 